\title{
Virtual reality rehabilitation following total knee arthroplasty: a systematic review and meta-analysis of randomized controlled trials
}

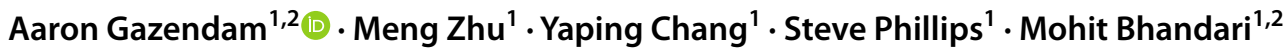 \\ Received: 16 November 2021 / Accepted: 27 January 2022 / Published online: 19 February 2022 \\ (c) The Author(s) under exclusive licence to European Society of Sports Traumatology, Knee Surgery, Arthroscopy (ESSKA) 2022
}

\begin{abstract}
Purpose The use of virtual reality (VR) based rehabilitation has increased substantially within orthopedic surgery, particularly in the field of total knee arthroplasty (TKA). The objective of this systematic review and meta-analysis was to compare patient-reported outcomes and cost analyses from randomized controlled trials (RCT) utilizing VR-based rehabilitation in patients following TKA.

Methods MEDLINE, EMBASE, and Cochrane databases were searched for RCTs involving VR-based rehabilitation following TKA. Quantitative synthesis was conducted for pain scores and functional outcomes. Narrative outcomes were reported for results not amenable to quantitative synthesis.

Results A total of 9 RCTs with 835 patients were included with follow-up ranging from 10 days to 6 months postoperatively. No differences in pain scores were demonstrated between VR-based and traditional rehabilitation at 2 weeks and 3 months postoperatively. VR-based rehabilitation demonstrated improved functional outcomes at 12 weeks $(n=353)$ postoperatively [mean difference (MD) - 3.32, 95\% confidence interval (CI) -5.20 to -1.45 , moderate certainty evidence] and 6 months ( $n=66$ ) postoperatively [MD $-4.75,95 \% \mathrm{CI}-6.69$ to -2.81 , low certainty evidence], compared to traditional rehabilitation. One trial demonstrated significant cost savings with the use of VR-based rehabilitation.

Conclusions VR-based rehabilitation for patients undergoing TKA represents an evolving field that may have advantages over traditional therapy for some patients. The current review is limited by the low quality of evidence in the literature. This is a rapidly evolving field with more trials needed to determine the impact of VR-based rehabilitation on patients undergoing TKA.
\end{abstract}

Level of evidence Level I; meta-analysis of randomized controlled trials.

Keywords Total knee arthroplasty $\cdot$ Virtual reality $\cdot$ Rehabilitation $\cdot$ Telemedicine $\cdot$ Telerehabilitation

\section{Introduction}

Physical rehabilitation following total knee arthroplasty (TKA) has been widely implemented and has been shown to improve early functional outcomes and patient satisfaction [25]. Post-hospitalization rehabilitation represents a significant proportion of the overall cost of TKAs and alternative

Aaron Gazendam and Meng Zhu denotes equal contribution.

Aaron Gazendam

aaron.gazendam@gmail.com

1 OrthoEvidence, Burlington, ON, Canada

2 Division of Orthopaedics, Department of Surgery, McMaster University, St. Joseph's Hospital, Room G522, 50 Charlton Avenue East, Hamilton, ON L8N 4A6, Canada delivery models have been explored [19]. In recent years, telerehabilitation has been proposed as an efficacious and cost-effective alternative to traditional in-person approaches $[20,21]$. A meta-analysis has demonstrated that telerehabilitation results in similar pain relief and functional outcomes when compared to face-to-face rehabilitation [12].

Virtual reality (VR) represents a core of technology which enables its users to be fully immersed in a simulated world and feel a sense of actual presence by providing multimodal stimuli [17]. The use of VR-based rehabilitation has increased substantially within orthopedic surgery and TKA in particular. A systematic review done by Blasco et al. (2019) examined evidence from randomized controlled trials (RCTs) published before January 2018 on the efficacy of VR-based rehabilitation in TKA but was only able to conduct a narrative summary [2]. A number of RCTs have been 
published since the release of the systematic review and an up-to-date systematic review and meta-analysis is needed and will be useful for clinicians and researchers $[1,8,12]$.

Several VR-based protocols have been proposed for rehabilitation following TKA. Early protocols utilized established interactive video games such as the Nintendo Wii ${ }^{\mathrm{TM}}$ as an adjunct to traditional therapy [5]. Advances in technology have allowed for more elaborate VR-based therapy with 3-dimensional tracking technology and digitally simulated coaches to demonstrate and provide immediate feedback on exercise quality [1]. VR therapy has been introduced as an adjunct to formal in-person therapy or as the primary mode of rehabilitation with telehealth therapists monitoring patients' progress in an asynchronous fashion based on the feedback from the VR platform $[1,5]$.

The purpose of this systematic review and meta-analysis was to analyze pain scores, functional outcomes and cost analyses from randomized controlled trials utilizing VRbased rehabilitation in patients following TKA. We hypothesize that VR-based rehabilitation will result in similar pain and functional outcomes with potential cost savings when compared to traditional rehabilitation.

\section{Methods}

This systematic review and meta-analysis was conducted in accordance with the PRISMA (Preferred Reporting Items for Systematic Reviews and Meta-Analyses) guidelines for conducting and reporting systematic reviews [22].

\section{Literature search}

Ovid MEDLINE, Ovid EMBASE, and Cochrane Controlled Register of Trials (CENTRAL) were searched for potentially eligible studies from inception to October 25, 2021. Both index terms and free-text terms regarding to "virtual reality" and "TKA" were searched and the results were filtered for RCTs. In addition, the reference lists of related systematic reviews were cross-referenced to identify eligible studies. The complete search strategy is available in the Appendix 1.

\section{Assessment of study eligibility}

The inclusion and exclusion criteria were defined a priori. Eligible studies were RCTs investigating a rehabilitation program involving a VR tool; defined as hardware/software devices creating a simulated environment for adult patients who have undergone TKA to interact with [16]. Only studies published in English were included. Non-randomized or quasi-randomized trials were excluded. Studies that included other joints or non-arthroplasty interventions of the knee were excluded. Studies that did not report functional outcomes or pain scores were included in the narrative review but excluded from the quantitative synthesis.

\section{Study screening and data extraction}

Two independent reviewers conducted study screening, inclusion, and extraction. Any disagreement between the two reviewers was resolved by consulting a third reviewer. The extracted data included study characteristics, rehabilitation protocol characteristics, patient demographics, costeffectiveness analyses, pain scores and functional outcomes. The pain score included in this review was the visual analog scale (VAS). The functional outcomes included in the quantitative synthesis were the Western Ontario and McMaster Universities Osteoarthritis Index (WOMAC) and the Knee Injury and Osteoarthritis Outcome Score (KOOS). Functional outcome data was extracted for the following time points; $<6$ weeks postoperatively, 12 weeks postoperatively; 6 months postoperatively. Pain scores were extracted at 2 weeks and 3 months postoperatively.

\section{Study appraisal and evidence synthesis}

Risk of bias and quality of evidence were determined using the Cochrane risk of bias 2 tool and the GRADE (Grading of Recommendations, Assessment, Development and Evaluations) approach, respectively [29, 32]. Quantitative synthesis was conducted using Review Manager (RevMan) 5 for pain outcomes measured by the VAS and functional outcomes. As per the guidelines set out by the GRADE all scores for each measured outcome were converted to a common scale [9]. A disease specific index (DSI) which incorporates the WOMAC and the KOOS was created [14, 27]. A meta-analysis using a random-effects model was performed and generated forest plots. Mean difference (MD) with 95\% confidence intervals (CI) were calculated between groups for both DSI and VAS scores and reported accordingly. $\mathrm{I}^{2}$ tests were used to assess heterogeneity across studies. Narrative summary of evidence was also provided. A $p$-value of $<0.05$ was considered statistically significant for all outcomes.

\section{Results}

\section{Literature search}

The results of the search are depicted in Fig. 1. After title, abstract and full text review, 9 RCTs with 835 patients randomized to VR-based telerehabilitation vs. traditional rehabilitation following TKA were identified and included in the final review [1, 4, 5, 8, 10, 13, 24, 26, 35]. 


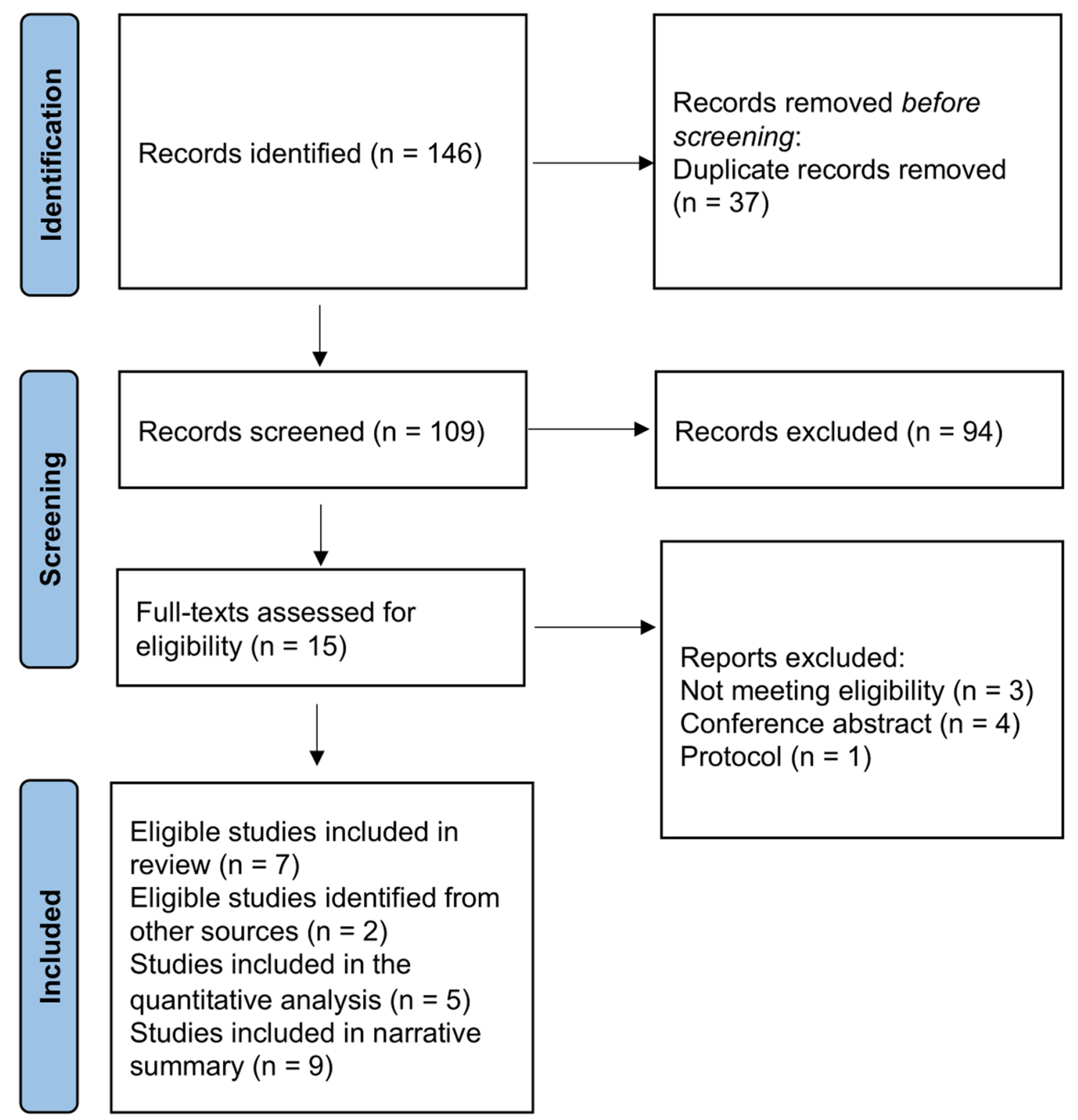

Fig. 1 PRISMA diagram

\section{Characteristics of included studies}

The characteristics of the included studies are described in Table 1. All patients underwent primary TKA. The median sample size was 50 (range 26-306). The median age of included patients was 68.5 (IQR; 66.9-72.7) years. Of the 835 patients, 299 (36\%) were male. Follow-up ranged from 10 days postoperatively to 6 months postoperatively.

\section{Study quality}

The risk of bias summary is available in the Appendix 2 . Overall, the highest risk of bias was due to concerns regarding the blinding of participants and personnel. Utilizing the GRADE approach, the quality of evidence was rated "very low" to "moderate" (Table 2).

\section{Pain scores}

Postoperative pain was evaluated using the VAS pain scores (Fig. 2). In studies evaluating pain scores in within 2 weeks following surgery $(n=282)$, no differences in pain scores were identified between the two groups $[8,13,24]$. Similarly, in studies evaluating pain scores $>3$ months from surgery $(n=133)$, no differences in pain scores were found between groups [24] (Fig. 2). 


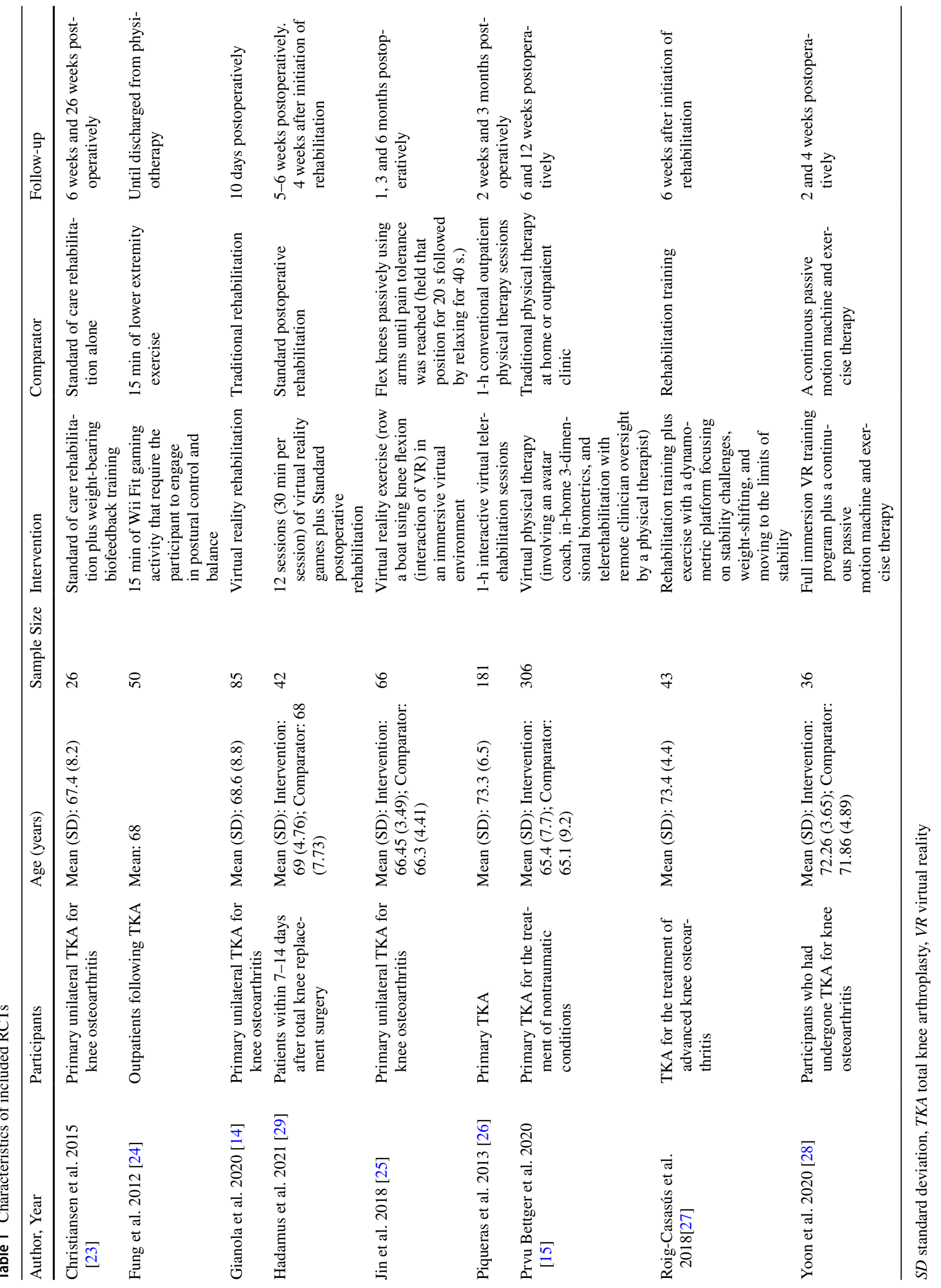


Table 2 GRADE Quality of Evidence

\begin{tabular}{lll}
\hline & Statistical Significance & Quality of Evidence \\
\hline VAS pain $(\leq 2$ weeks after TKA) & No & Very Low \\
VAS pain (3 months after TKA) & No & Very Low \\
Disease specific index ( $\leq 6$ weeks after TKA) & No & Very Low \\
Disease specific index (12 weeks after TKA) & Yes & Moderate \\
Disease specific index (6 months after TKA) & Yes & Low \\
\hline
\end{tabular}

GRADE Grading of Recommendations, Assessment, Development and Evaluations, VAS visual analog scale, TKA total knee arthroplasty

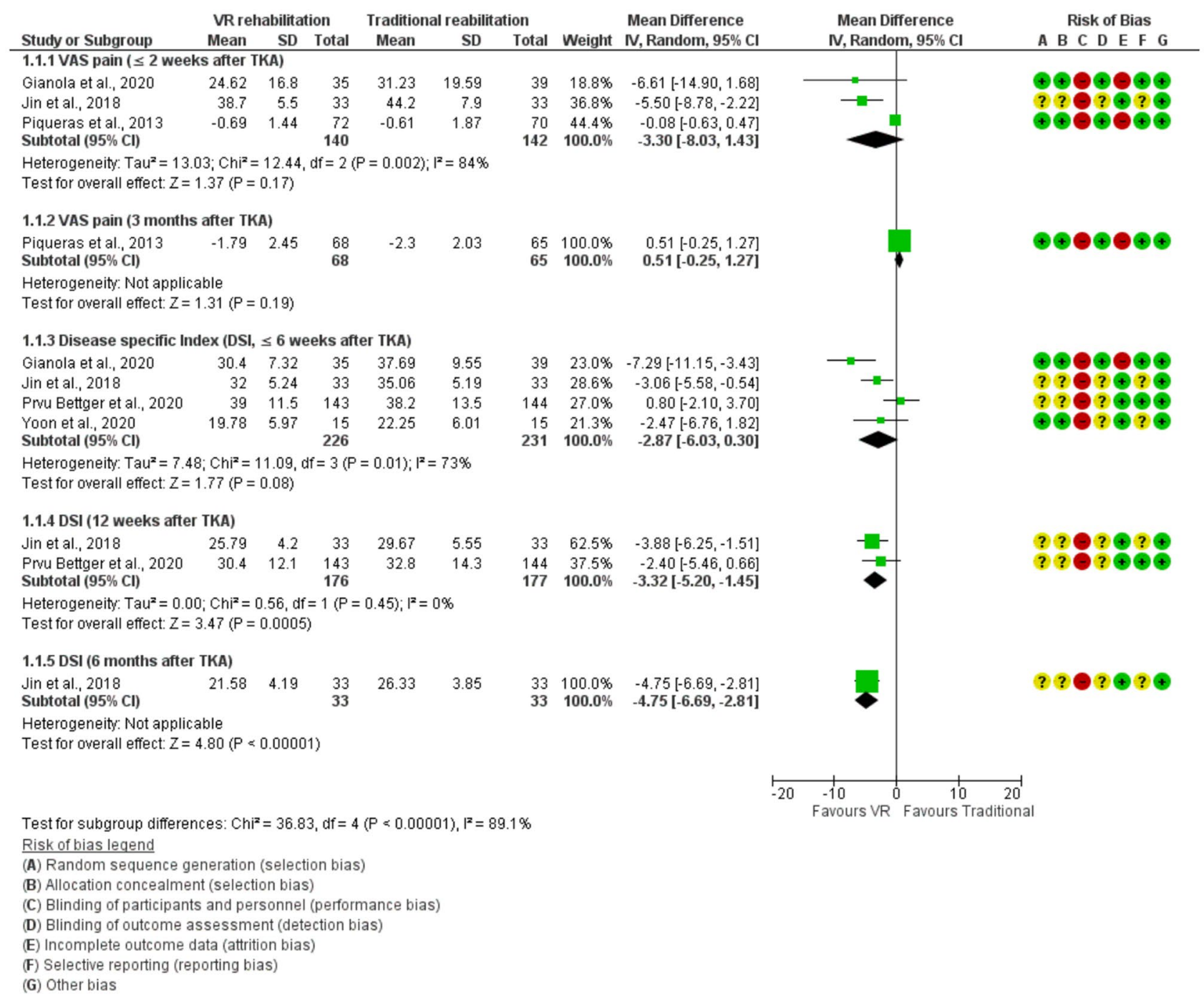

Fig. 2 Forest charts depicting pain and disease specific indices. $C$ confidence interval, VAS visual analog scale, TKA total knee arthroplasty, DSI disease specific index, $V R$ virtual reality

\section{Disease specific index}

The disease specific scores utilized in the included studies were the WOMAC and the KOOS. Four studies $(n=457)$ evaluated DSI within the 6 -week postoperative period
(Fig. 2) [1, 8, 13, 35]. At this time point, no significant differences were demonstrated. VR-based rehabilitation made statistically significant improvements in DSI at 12 weeks $(n=353)$ postoperatively [mean difference (MD) -3.32 , $95 \%$ confidence interval $(\mathrm{CI})-5.20$ to -1.45$]$ and 6 months 
Table 3 Narrative summary of key findings from included RCTs

\begin{tabular}{|c|c|}
\hline Author, Year & Key Findings \\
\hline Christiansen et al. 2015 [23] & $\begin{array}{l}\text { A 6-week of weight-bearing biofeedback training plus standard of care rehabilitation resulted in an improved } \\
\text { five times sit-to-stand test times and an increase in knee extension moments during gait, compared to standard } \\
\text { of care rehabilitation alone. However, the intervention did not improve functional weight-bearing symmetry or } \\
\text { knee extension moments during the five times sit-to-stand test }\end{array}$ \\
\hline Fung et al. 2012 [24] & $\begin{array}{l}\text { After examining the percentage change from study enrollment to discharge, the authors found no statistically } \\
\text { significant difference in outcomes such as pain, knee flexion and extension, walking speed, timed standing } \\
\text { tasks, Lower Extremity Functional Scale, Activity-specific Balance Confidence Scale, or patient satisfaction, } \\
\text { between VR-based rehabilitation (Nitendo Wii Fit gaming activity) and traditional rehabilitation (lower extrem- } \\
\text { ity exercise) }\end{array}$ \\
\hline Gianola et al. 2020 [14] & $\begin{array}{l}\text { VR-based rehabilitation resulted in a significant improvement in the global proprioception, but did not improve } \\
\text { outcomes such as pain and function, compared to traditional rehabilitation }\end{array}$ \\
\hline Hadamus et al. 2021 [29] & $\begin{array}{l}\text { No significant improvements in the postural stability parameters assessed were observed in neither the VR-based } \\
\text { rehabilitation group nor the standard postoperative rehabilitation group }\end{array}$ \\
\hline Jin et al. 2018 [25] & $\begin{array}{l}\text { Compared to traditional rehabilitation, VR-based rehabilitation significantly reduced postoperative pain at 3, } 5 \text {, } \\
\text { and } 7 \text { days post TKA, improved Western Ontario and McMaster University osteoarthritis index (WOMAC) } \\
\text { as well as Hospital for Special Surgery knee score (HSS) at 1,3,6 months post TKA. Knee range of motion } \\
\text { was significantly higher in the VR-based rehabilitation group than traditional rehabilitation group at 3, 7, and } \\
14 \text { days post TKA }\end{array}$ \\
\hline Piqueras et al. 2013 [26] & $\begin{array}{l}\text { VR rehabilitation (a 2-week interactive virtual telerehabilitation), which achieved improvements similar to the } \\
\text { traditional rehabilitation group did in most outcome variables (e.g., active knee flexion, visual analog pain), } \\
\text { was non-inferior to traditional face -to-face rehabilitation }\end{array}$ \\
\hline Prvu Bettger et al. 2020 [15] & $\begin{array}{l}\text { Virtual physical therapy with telerehabilitation significantly reduced the healthcare cost at } 12 \text { weeks after dis- } \\
\text { charge and was non-inferior to traditional physical therapy in terms of Knee injury and Osteoarthritis Outcome } \\
\text { Score (KOOS), knee extension, knee flexion, gait speed, pain, and hospital readmission. Patients undergoing } \\
\text { virtual rehabilitation had significantly better adherence to exercise }\end{array}$ \\
\hline Roig-Casasús et al. 2018 [27] & $\begin{array}{l}\text { VR rehabilitation improved balance performance according to the Berg Balance Scale and Functional Reach Test } \\
\text { compared to traditional rehabilitation }\end{array}$ \\
\hline Yoon et al. 2020 [28] & VR rehabilitation yielded better early balance ability and knee function, compared to the control group \\
\hline
\end{tabular}

$(n=66)$ postoperatively [MD $-4.75,95 \% \mathrm{CI}-6.69$ to

$-2.81]$, compared to traditional rehabilitation $[1,13]$.

\section{Narrative results}

A complete summary of key outcomes not amenable to statistical synthesis are outlined in Table 3. Only one trial evaluated the cost-effectiveness of VR-based rehabilitation. Prvu Bettger et al. demonstrated that VR-based rehabilitation costs significantly less than traditional rehabilitation [median in United States dollars: 1050 vs. 2805, $p<0.001$ ] at 12 weeks after surgery [1].

\section{Discussion}

The most important findings of the present study were that VR-based rehabilitation had significantly better patientreported outcome scores at 3 and 6 months postoperatively and similar improvements in postoperative pain when compared to traditional rehabilitation protocols.

Postoperative rehabilitation aiming at functional restoration and pain relief is an essential and critical part of the recovery process [25]. Studies have shown that postoperative rehabilitation could be beneficial to patients following TKA, including shortening hospital stays and reducing complications [3]. Telerehabilitation, including VR-based therapy, has the potential to address barriers to access including therapist availability and presence of therapy centers within a reasonable distance. The projected increase in TKA is expected to outpace the availability of physical therapists [11]. VR-based therapy has the potential to reduce that burden as therapists can be utilized asynchronously and provide synchronous care when progress stalls [1]. VR-based therapy can be performed from home, avoiding the need for transportation to a therapy center, making it a potential time and cost-saving measure [20]. This is particularly valuable in the immediate postoperative period when patients are unable to drive themselves to appointments or with patients located in rural areas.

A critical aspect of postoperative therapy is patient adherence. Lack of adherence to postoperative rehabilitation protocols has been linked to increased pain, stiffness and weakness following TKA [25]. Factors affecting adherence to exercise adherence in this population include timing, transportation, access to exercise equipment and cost of physiotherapy sessions [18, 23, 34]. Given that VR-based telerehabilitation has the opportunity to address many of 
these barriers, there may be an opportunity for improved exercise adherence postoperatively. Bettger el al (2020) demonstrated that patients undergoing VR-based telerehabilitation had significantly greater adherence to their prescribed protocols than patients enrolled in traditional therapy which may have played a role in the improved functional outcomes observed [1].

One of the driving forces in the shift towards telerehabilitation as a whole is the potential cost savings at both a patient and systems level [33]. Telerehabilitation has demonstrated cost savings compared to face-to-face therapy, particularly when travel costs are considered [6,33]. Although telerehabilitation addresses geographic and transportation barriers, the cost and availability of therapists remains a barrier to access. VR-based rehabilitation has the potential to eliminate these barriers as patients can access and perform therapy from home without requiring an available therapist. Only Bettger et al. (2020) reported the cost and safety outcomes among the included RCTs [1]. They demonstrated that VR-based rehabilitation costs significantly less than traditional rehabilitation and reported that significantly fewer patients receiving VR-based rehabilitation were re-hospitalized, compared to those who had traditional rehabilitation. Future trials should include cost analyses and comparisons between VR-based telerehabilitation and therapist based telerehabilitation.

Finally, the ongoing Covid-19 pandemic has necessitated the rapid adoption and integration of digital health tools including VR-based rehabilitation [7, 15]. VR-based rehabilitation has been implemented across disciplines to provide effective rehabilitation programs while conferring to social distancing measures and minimizing risk for patients. Within the pandemic, VR-based rehabilitation has been successfully implemented for patients with cognitive disorders, chronic obstructive pulmonary disorder, chronic low back pain and Covid-19 itself [7, 28, 31]. A review by Singh et al. (2020) stated that VR technology could decrease the risk of contracting COVID-19 by reducing the in-person contact between healthcare personnel and patients, and should be considered as a "complementary medical/healthcare edify tool [that] will enhance the execution of medical deliverables." [30]

This review is limited by the current available literature. There were significant variations in both the VR and standard protocols with respect to type of exercises and duration of rehabilitation. This variability makes it more difficult to make meaningful conclusions from synthesized data in a meta-analysis. Given that no trials have analyzed different VR-rehabilitation protocols, the optimal strategy and protocol remains unknown. Additionally, we rated the quality of evidence as moderate to very low, indicating that current evidence is inadequate to allow a clear conclusion. Small sample size in the meta-analysis is one of the main concerns.

\section{Conclusions}

VR-based rehabilitation for patients undergoing TKA represents an evolving field that may have advantages over traditional therapy for some patients. VR-based rehabilitation has the potential to reduce costs at both the healthcare and patient level, increase exercise adherence and reduce barriers to accessing postoperative therapy following TKA. However, the review is limited by the available of high-quality evidence and variability in the rehabilitation protocols. Future research is required to confirm the results of the current study and to evaluate the optimal VR-rehabilitation protocol.

Supplementary Information The online version contains supplementary material available at https://doi.org/10.1007/s00167-022-06910-x.

Funding This research did not receive any specific grant from funding agencies in the public, commercial, or not-for-profit sectors.

\section{Declarations}

Conflict of interest MB reports personal fees from Pendopharm, Bioventus and Accumed, outside the submitted work. All other authors (AG, MZ, YC, SP) report no conflicts of interest.

Ethical approval Not applicable.

\section{References}

1. Bettger JP, Green CL, Holmes DN, Chokshi A, Mather RC III, Hoch BT, de Leon AJ, Aluisio F, Seyler TM, Del Gaizo DJ (2020) Effects of virtual exercise rehabilitation in-home therapy compared with traditional care after total knee arthroplasty: VERITAS, a randomized controlled trial. J Bone Joint Surg Am 102:101-109

2. Blasco J, Igual-Camacho C, Blasco M, Anton-Anton V, OrtizLluecalvaro, Roig-Casasus S, (2021) The efficacy of virtual reality tools for total knee replacement rehabilitation: a systematic review. Physiother Theory Pract 37:682-692

3. Castrodad IMD, Recai TM, Abraham MM, Etcheson JI, Mohamed NS, Edalatpour A, Delanois RE (2019) Rehabilitation protocols following total knee arthroplasty: a review of study designs and outcome measures. Ann Transl Med 7:S255

4. Christiansen CL, Bade MJ, Davidson BS, Dayton MR, StevensLapsley JE (2015) Effects of weight-bearing biofeedback training on functional movement patterns following total knee arthroplasty: a randomized controlled trial. J Orthop Sports Phys Ther 45:647-655

5. Fung V, Ho A, Shaffer J, Chung E, Gomez M (2012) Use of Nintendo Wii Fit ${ }^{\mathrm{TM}}$ in the rehabilitation of outpatients following total knee replacement: a preliminary randomised controlled trial. Physiotherapy 98:183-188

6. Fusco F, Turchetti G (2016) Telerehabilitation after total knee replacement in Italy: cost-effectiveness and cost-utility analysis of a mixed telerehabilitation-standard rehabilitation programme compared with usual care. BMJ Open 6:e009964 
7. Garcia LM, Birckhead BJ, Krishnamurthy P, Sackman J, Mackey IG, Louis RG, Salmasi V, Maddox T, Darnall BD (2021) An 8-Week Self-Administered At-Home Behavioral Skills-Based Virtual Reality Program for Chronic Low Back Pain: DoubleBlind, Randomized, Placebo-Controlled Trial Conducted During COVID-19. J Med Internet Res 23:e26292

8. Gianola S, Stucovitz E, Castellini G, Mascali M, Vanni F, Tramacere I, Banfi G, Tornese D (2020) Effects of early virtual reality-based rehabilitation in patients with total knee arthroplasty: a randomized controlled trial. Medicine (Baltimore) 99

9. Guyatt GH, Thorlund K, Oxman AD, Walter SD, Patrick D, Furukawa TA, Johnston BC, Karanicolas P, Akl EA, Vist G (2013) GRADE guidelines: 13. Preparing summary of findings tables and evidence profiles - continuous outcomes. J Clin Epidemiol 66:173-183

10. Hadamus A, Białoszewski D, Błażkiewicz M, Kowalska AJ, Urbaniak E, Wydra KT, Wiaderna K, Boratyński R, Kobza A, Marczyński W (2021) Assessment of the effectiveness of rehabilitation after total knee replacement surgery using sample entropy and classical measures of body balance. Entropy (Basel) 23:164

11. Inacio MCS, Paxton EW, Graves SE, Namba RS, Nemes S (2017) Projected increase in total knee arthroplasty in the United States-an alternative projection model. Osteoarthritis Cartilage 25:1797-1803

12. Jiang S, Xiang J, Gao X, Guo K, Liu B (2018) The comparison of telerehabilitation and face-to-face rehabilitation after total knee arthroplasty: a systematic review and meta-analysis. J Telemed Telecare 24:257-262

13. Jin C, Feng Y, Ni Y, Shan Z (2018) Virtual reality intervention in postoperative rehabilitation after total knee arthroplasty: a prospective and randomized controlled clinical trial. Int J Clin Exp Med 11:6119-6124

14. Jinks C, Jordan K, Croft P (2002) Measuring the population impact of knee pain and disability with the Western Ontario and McMaster Universities Osteoarthritis Index (WOMAC). Pain 100:55-64

15. Karasavvidis T, Hirschmann MT, Kort NP, Terzidis I, Totlis T (2020) Home-based management of knee osteoarthritis during COVID-19 pandemic: literature review and evidence-based recommendations. J Exp Orthop 7:52

16. Keshner EA (2004) Virtual reality and physical rehabilitation: a new toy or a new research and rehabilitation tool? BMC Med $14: 215$

17. Li A, Montaño Z, Chen VJ, Gold JI (2011) Virtual reality and pain management: current trends and future directions. Pain Manag $1: 147-157$

18. Marks R (2014) Self-efficacy and arthritis disability: An updated synthesis of the evidence base and its relevance to optimal patient care. Health Psychol Open 1:2055102914564582

19. Mechanic R (2014) Post-acute care-the next frontier for controlling Medicare spending. N Engl J Med 370:692-694

20. Moffet H, Tousignant M, Nadeau S, Mérette C, Boissy P, Corriveau H, Marquis F, Cabana F, Belzile ÉL, Ranger P (2017) Patient satisfaction with in-home telerehabilitation after total knee arthroplasty: results from a randomized controlled trial. Telemed J E Health 23:80-87

21. Moffet H, Tousignant M, Nadeau S, Mérette C, Boissy P, Corriveau $\mathrm{H}$, Marquis F, Cabana F, Ranger P, Belzile ÉL, Dimentberg $\mathrm{R}$ (2015) In-home telerehabilitation compared with face-to-face rehabilitation after total knee arthroplasty: a noninferiority randomized controlled trial. J Bone Joint Surg Am 97:1129-1141
22. Page MJ, McKenzie JE, Bossuyt PM, Boutron I, Hoffmann TC, Mulrow CD, Shamseer L, Tetzlaff JM, Akl EA, Brennan SE (2021) The PRISMA 2020 statement: an updated guideline for reporting systematic reviews. BMJ 372

23. Petursdottir U, Arnadottir SA, Halldorsdottir S (2010) Facilitators and barriers to exercising among people with osteoarthritis: a phenomenological study. Phys Ther 90:1014-1025

24. Piqueras M, Marco E, Coll M, Escalada F, Ballester A, Cinca C, Belmonte R, Muniesa JM (2013) Effectiveness of an interactive virtual telerehabilitation system in patients after total knee arthroplasty: a randomized controlled trial. J Rehabil Med 45:392-396

25. Pozzi F, Snyder-Mackler L, Zeni J (2013) Physical exercise after knee arthroplasty: a systematic review of controlled trials. Eur J Phys Rehabil Med 49:877

26. Roig-Casasús S, Blasco JM, López-Bueno L, Blasco-Igual MC (2018) Balance training with a dynamometric platform following total knee replacement: a randomized controlled trial. J Geriatr Phys Ther 41:204-209

27. Roos EM, Roos HP, Lohmander LS, Ekdahl C, Beynnon BD (1998) Knee Injury and Osteoarthritis Outcome Score (KOOS) development of a self-administered outcome measure. J Orthop Sports Phys Ther 28:88-96

28. Rutkowski S (2021) Management challenges in chronic obstructive pulmonary disease in the covid-19 pandemic: telehealth and virtual reality. J Clin Med 10:1261

29. Schünemann H, Brożek J, Guyatt G, Oxman A (2015) GRADE handbook. 2013.

30. Singh RP, Javaid M, Kataria R, Tyagi M, Haleem A, Suman R (2020) Significant applications of virtual reality for COVID-19 pandemic. Diabetes Metab Snydr 14:661-664

31. Smits M, Staal JB, Van Goor H (2020) Could Virtual Reality play a role in the rehabilitation after COVID-19 infection? BMJ Open Sport Exerc Med 6:e000943

32. Sterne JAC, Savović J, Page MJ, Elbers RG, Blencowe NS, Boutron I, Cates CJ, Cheng H-Y, Corbett MS, Eldridge SM, Emberson JR, Hernán MA, Hopewell S, Hróbjartsson A, Junqueira DR, Jüni P, Kirkham JJ, Lasserson T, Li T, McAleenan A, Reeves BC, Shepperd S, Shrier I, Stewart LA, Tilling K, White IR, Whiting PF, Higgins JPT (2019) RoB 2: a revised tool for assessing risk of bias in randomised trials. BMJ 366:14898

33. Tousignant M, Moffet H, Nadeau S, Mérette C, Boissy P, Corriveau $\mathrm{H}$, Marquis F, Cabana F, Ranger P, Belzile ÉL (2015) Cost analysis of in-home telerehabilitation for post-knee arthroplasty. J Med Internet Res 17:e3844

34. Webster F, Perruccio AV, Jenkinson R, Jaglal S, Schemitsch E, Waddell JP, Venkataramanan V, Bytautas J, Davis AM (2015) Understanding why people do or do not engage in activities following total joint replacement: a longitudinal qualitative study. Osteoarthritis Cartilage 23:860-867

35. Yoon S, Son H (2020) Effects of full immersion virtual reality training on balance and knee function in total knee replacement patients: a randomized controlled study. J Mech Med Biol 20:2040007

Publisher's Note Springer Nature remains neutral with regard to jurisdictional claims in published maps and institutional affiliations. 